\title{
Dynamic Incentive Mechanism of Multitask Cooperation in Logistics Supply Chain
}

\author{
Song Wang, ${ }^{1}$ Yang Zhao $\left(D,{ }^{2}\right.$ Lanfeng Liu, ${ }^{3}$ and Fuhua Huang ${ }^{1}$ \\ ${ }^{1}$ College of Business Administration, Hunan University of Technology and Business, Hunan, China \\ ${ }^{2}$ School of Business, Suzhou University of Science and Technology, Suzhou, China \\ ${ }^{3}$ School of Business Administration, Anhui University, Anhui, China \\ Correspondence should be addressed to Yang Zhao; zhaoyang@mail.usts.edu.cn
}

Received 20 October 2020; Accepted 15 February 2021; Published 8 March 2021

Academic Editor: Maojun Zhang

Copyright ( $\odot 2021$ Song Wang et al. This is an open access article distributed under the Creative Commons Attribution License, which permits unrestricted use, distribution, and reproduction in any medium, provided the original work is properly cited.

This paper studies the incentive mechanism of multitask cooperation in logistics service supply chain (LSSC) by building a dynamic incentive model. Research shows the following: (1) the implicit reputation in dynamic cooperation can effectively improve system incentive effectiveness; (2) the difference in the contribution of different logistics cooperation to the performance of the LSSC has a significant impact on the incentive effect; (3) when two kinds of cooperation tasks have complementary relationships, both the LSP's choice of logistics tasks and the incentives will simultaneously act on the two types of cooperation tasks; and (4) the antirisk degree of LSI and the environment uncertainty will also have a certain impact on the incentive effect of the LSSC. Therefore, the LSI should comprehensively consider the requirements of logistics cooperation and the risk tolerance of LSI to be chosen.

\section{Introduction}

In logistics supply chain (LSSC), logistics service integrator (LSI) occupies a dominant position and faces customers directly, so it can accurately determine the potential needs of customers. Logistics service providers (LSPs), as professional service provider in specific logistics activities, can transform the service demand judgment of LSI into valuable business services, ultimately enhancing the value of LSSC. The LSSC value relationship led by LSI through logistics outsourcing and resource cooperation with LSP is a typical agency relationship.

LSSC is an integrated supply chain that integrates multiple logistics service capabilities to jointly create customer value. In LSSC, the leading LSI, as designers and providers of integrated logistics service solutions, has high requirements for cooperating in different types of aspects. The differences in different types of logistics services and the conflict in the LSP's investment in different logistics services have caused great impact on the LSSC incentive efficiency. So, the theoretical framework of principal agent and the mathematical method of game theory are introduced to study the problem of revenue sharing in the supply chain and to coordinate the relationship between principal and agent, eventually coordinating the whole supply chain.

At the same time, due to the high complexity and dynamic nature of integrated logistics service, the large number of logistics service requires LSI, LSP, and client companies to cooperate in multiple phases. With the asymmetry of upstream and downstream information, this kind of crossphase dynamic cooperation and the inconsistency in the interest of cooperation partners have greatly increased the difficulty in system coordination. Therefore, how to establish a good principal-agent relationship in LSSC cooperation and implement effective incentive mechanisms to improve system operation capabilities and overall performance has become an important issue in LSSC practice.

Many scholars have conducted research in multitask incentive and dynamic incentive $[1,2]$ and have achieved fruitful results in the logistics supply chain [3]. Lau et al. [4] in response to different market needs, the change in retailer risk cost, supplier incentive cost, and general agent cost in 
two-stage supply chain and their influence on the maximum profit of supply chain are studied by the condition of complete or incomplete information. Albert et al. [5] aiming at the supply chain coordination problem under the condition of incomplete information proposed a commitmentbased incentive mechanism. Through the design of this mechanism, information sharing and mutual cooperation among supply chain members are promoted, so as to increase the trust of supply chain member companies and supply chain overall benefits. Ngyuyen and Poundon [6] and Krishnan and Winter [7] studied the dynamic incentive problem in which the agent information parameters are unrelated across stages and the principal model implements full commitment.

Based on the assumptions of different income models and information structures, some scholars made a comparative analysis of the single-stage static incentive problem and the cross-stage dynamic incentive problem of supply chain [8-11]. Yingying et al. [12] analyzed the influencing factors and decision-making mechanism of open collaborative innovation between enterprises under the condition of no incentive and punishment mechanism and the introduction of different incentive and punishment mechanisms. The study found that the cost sharing between enterprises was excessive. Benefits and the knowledge spillover effect when one party "free rides" have an impact on the enterprise's open collaborative innovation strategy. After introducing the incentive and punishment mechanisms $[13,14]$, it is further discovered that the rewards and punishments and the number of cooperation have a positive impact on the promotion of enterprise open collaborative innovation. Cost-sharing coefficient is negatively related to it.

According to the different nature of different logistics cooperation, cooperation tasks can be divided into two types: regular logistics cooperation tasks such as sharing complementary logistics resource and value-added logistics cooperation tasks. Furthermore, from the long-run perspective, a dynamic incentive model under multitask cooperation is constructed to simulate the cross-phase cooperation of LSSC. This paper focuses on the following issues: how does the implicit reputation effect affect the behavior of partners in the dynamic cooperation course of LSSC? How does the relevance of the two types of cooperation tasks and the LSP antirisk degree affect incentive effectiveness? According to the above research, this paper may provide a certain reference for the design and implementation of the incentive mechanism in the operation of LSSC.

\section{Parameter Setting and Model Construction}

2.1. Problem Description and Parameter Setting. In addition to regular tasks, LSI puts forward some more professional logistics service to be accomplished by LSP, such as providing logistics information services. According to the nature of logistics service cooperation, the former is called a regular task, and the latter is called a value-added task. The integration of value-added logistics service cooperation over regular cooperation is an important nature of LSSC which is distinguished from merely basic logistics cooperation. Further investigation shows that, due to high complexity of integrated two types of logistics service cooperation, a great deal of cooperation requires continuous multiphase to play games and realize cooperation between LSI and LSP.

In order to describe the strategic interaction between the LSI and LSP in the cooperation of the LSSC, some operating parameters need to be set.

2.1.1. LSSC Income Function Setting. LSPs are commissioned by LSI to engage in two types of logistics service cooperation tasks, the level of which is set to $L_{1 t}$ and $L_{2 t}$, respectively (Table 1 ).

Considering the differences in the impact of the two types of cooperation tasks on the output of the whole system, it is further assumed that $\eta$ and $\varepsilon_{t}$ are normally independent distributions: $\quad \eta \sim N\left(0, \varphi \sigma^{2}\right), \quad \varepsilon_{t} \sim N\left(0,(1-\varphi) \sigma^{2}\right)$, and $\varepsilon_{1}$ and $\varepsilon_{2}$ are independent of each other, namely, $\operatorname{cov}\left(\varepsilon_{1}, \varepsilon_{2}\right)=0$, and $\eta$ and $\varepsilon_{t}$ are not correlated $[15,16]$.

According to the definition of task relevance and the specific characteristics of the two types of cooperation tasks, set coefficient of the impact of value-added logistics service cooperation task on the output of the system to $r(r \in[-1,1])$ (Table 2).

According to the definition of task relevance and the specific characteristics of the two types of cooperation tasks, $r<0$, that is, if the investment in the value-added logistics service cooperation task increases, the quality and efficiency of regular logistics service cooperation task will correspondingly raise, thereby reducing their marginal costs.

Assume that the effort cost function of LSP on two types of logistics cooperation tasks is

$$
I\left(L_{1 t}, L_{2 t}\right)=\frac{1}{2} \beta_{1} L_{1 t}^{2}+r \sqrt{\beta_{1} \beta_{2}} L_{1 t}^{2} L_{2 t}^{2}+\frac{1}{2} \beta_{2} L_{2 t}^{2} .
$$

Assume that the cooperation of the LSSC lasts for two phases, and the output capacity function of the cross-phase cooperation is

$$
y_{t}=L_{1 t}+\varnothing L_{2 t}+\eta+\varepsilon_{t}, \quad t=1,2 .
$$

Then, the income function of the LSI is $y_{t}-I\left(L_{1 t}, L_{2 t}\right)$.

2.1.2. LSSC Cooperation Function Setting. Regarding the principal-agent cooperation mechanism, Weitman [17] and Holmstrom [18] proved that the linear function is reasonable and can achieve optimal results in their literature, respectively. In this paper, linear functions are used as the cooperation mechanism model [19]. Set the linear cooperation incentive function provided by the LSP to the LSI as

$$
\pi_{t}\left(y_{t}\right)=a_{t}+b_{t}\left(y_{t}\right), \quad t=1,2
$$

The cross-phase cooperation incentives differ from single-phase static in the unobservable capabilities of LSP, so the LSI has to use the actual output $y_{1}$ of phase I to estimate $\mathrm{LSP}^{\prime}$ ability, so as to determine the incentive intensity to LSP in the phase II. 
TABle 1: Model index setting-1.

\begin{tabular}{|c|c|c|}
\hline & Index & Index description \\
\hline$y_{t}$ & System output of the LSSC & Represents the system output of the LSSC in the $t$ phase of cooperation \\
\hline$L_{1 t}$ & Efforts of I SP & Represents the efforts of LSP in regular logistics service cooperation task in the $t$ phase \\
\hline$L_{2 t}$ & Ellorts or LSP & Represents the effort of LSP in the value-added cooperation task in the $t$ phase \\
\hline$\eta$ & Logistics service capacity & Represents the logistics service capacity of the LSP \\
\hline$\varnothing$ & $\begin{array}{l}\text { Coefficient of the impact of } \\
\text { value-added }\end{array}$ & $\begin{array}{c}\text { Represents the coefficient of the impact of value-added logistics service cooperation task on the } \\
\text { output of the system }\end{array}$ \\
\hline$\varepsilon_{t}$ & Random factors & $\begin{array}{l}\text { Represents the random factors that affect the output of different tasks and also determines the degree } \\
\text { of observability of different tasks }\end{array}$ \\
\hline
\end{tabular}

TABLE 2: Coefficient of association $r$.

\begin{tabular}{|c|c|c|c|}
\hline Coefficient & Index description & Range & Sampling description \\
\hline$r$ & $\begin{array}{l}\text { Coefficient of the impact of value-added } \\
\text { logistics service cooperation task } \\
\text { on the output of the system }\end{array}$ & $r \in[-1,0)$ & $\begin{array}{l}\text { The two types of cooperation tasks had a complementary relationship; } \\
\text { that is, if the investment in the value-added logistics service } \\
\text { cooperation task increases, the quality and efficiency of regular logistics } \\
\text { service cooperation task will correspondingly raise, thereby reducing } \\
\text { their marginal costs }\end{array}$ \\
\hline & & $\begin{array}{l}r=0 \\
r \in(0,1]\end{array}$ & $\begin{array}{l}\text { The two types of cooperation tasks are independent of each other } \\
\text { The two types of cooperation tasks have a substitute relationship }\end{array}$ \\
\hline
\end{tabular}

On the contrary, LSP can influence the judgment of the LSI by determining the impact of the two service effort levels $\left(L_{11}, L_{21}\right)$ on system output $y_{1}$ in phase I. Therefore, the level of effort of the LSP in phase I not only affects their current income but also affects the income of the subsequent cooperation phase, thereby prompting the LSP to be responsible for its current cooperative behavior. This is the reputation effect in dynamic cooperation. This mechanism has improved the long-term cooperation performance of the LSSC to a certain extent.

Let $\varphi=\operatorname{var}(\eta) /\left(\operatorname{var}(\eta)+\operatorname{var}\left(\varepsilon_{t}\right)\right)$. Based on the above assumptions, according to rational expectations, we can get

$$
\begin{aligned}
E\left(y_{2} \mid y_{1}\right) & =\widehat{L}_{12}+\varphi\left(y_{1}-\widehat{L}_{11}-\varnothing \widehat{L}_{21}\right)+\varnothing \widehat{L}_{22}, \\
\operatorname{var}\left(y_{2} \mid y_{1}\right) & =\left(1-\varphi^{2}\right) \sigma^{2} \\
E\left(\eta \mid y_{1}\right) & =\varphi\left(y_{1}-\widehat{L}_{11}-\varnothing \widehat{L}_{21}\right) .
\end{aligned}
$$

2.1.3. The Utility Function of Both Parties of the LSSC Cooperation. Based on the risk, utility function can be described as follows: the LSI as the principal is risk-neutral, and the LSP is risk-averse, and their utility function has the invariable absolute risk aversion feature, that is, $U(\pi)=-e^{-\rho \pi}$, and $\rho$ is the absolute risk aversion measure $(\rho \in[-1,1]), \pi$ is the actual income of the LSP, and $i(i>0)$ is the discount rate. Subscript $A$ indicates the LSI, and subscript $C$ indicates the LSP. Set $\lambda_{A}$ and $\lambda_{C}$ as the altruistic coefficient of the LSI and LSP, respectively.

So the utility functions of the LSI and LSP are expressed as

$$
\begin{aligned}
& U_{A}=y_{1}-\pi_{1}+i\left(y_{2}-\pi_{2}\right), \\
& U_{C}=-\exp \left(-\rho\left\{\pi_{1}-I\left(L_{11}, L_{21}\right)+i\left[\pi_{2}-I\left(L_{12}, L_{22}\right)\right]\right\}\right) .
\end{aligned}
$$

The LSI and LSP utility functions with altruism are

$$
\begin{aligned}
U_{A}^{\prime}= & {\left[y_{1}-\pi_{1}+i\left(y_{2}-\pi_{2}\right)\right]+\lambda_{A} *\left(-\exp -\rho\left\{\pi_{1}-I\left(L_{11}, L_{21}\right)\right.\right.} \\
& \left.\left.+i\left[\pi_{2}-I\left(L_{12}, L_{22}\right)\right]\right\}\right), \\
U_{C}^{\prime}= & -\exp \left(-\rho\left\{\pi_{1}-I\left(L_{11}, L_{21}\right)+i\left[\pi_{2}-I\left(L_{12}, L_{22}\right)\right]\right\}\right) \\
& +\lambda_{c} *\left(\left[y_{1}-\pi_{1}+i\left(y_{2}-\pi_{2}\right)\right]\right) .
\end{aligned}
$$

The deterministic equivalent of the LSI with altruism and the deterministic equivalent of LSP are

$I E_{A}=E\left[y_{1}-\pi_{1}\left(y_{1}\right)\right]+i E\left[\left(y_{2}-\pi_{2}\left(y_{2}\right)\right)\right]+\lambda_{A} *\left(E\left[\pi_{1}\left(y_{1}\right)-I\left(L_{11}, L_{21}\right)\right]-\frac{1}{2} \rho \operatorname{var}\left(\pi_{1}+\delta \pi_{2}\right)+i E\left[\pi_{2}\left(y_{2}\right)-I\left(L_{12}, L_{22}\right)\right]\right)$

$I E_{C}=E\left[\pi_{1}\left(y_{1}\right)-I\left(L_{11}, L_{21}\right)\right]-\frac{1}{2} \rho \operatorname{var}\left(\pi_{1}+\delta \pi_{2}\right)+i E\left[\pi_{2}\left(y_{2}\right)-I\left(L_{12}, L_{22}\right)\right]+\lambda_{c} *\left(E\left[y_{1}-\pi_{1}\left(y_{1}\right)\right]+i E\left[\left(y_{2}-\pi_{2}\left(y_{2}\right)\right)\right]\right)$. 
$E\left[\pi_{1}\left(y_{1}\right)-I\left(L_{11}, L_{21}\right)\right]$ is the expected benefit of phase I, $i E\left[\pi_{2}\left(y_{2}\right)-I\left(L_{12}, L_{22}\right)\right]$ is the expected benefit of phase II, and $(1 / 2) \rho \operatorname{var}\left(\pi_{1}+\delta \pi_{2}\right)$ is the total risk cost of the LSP in two phases.

2.2. Cooperative Incentive Model Construction. The game sequence of cross-phase cooperation can be described as follows: in phase I, the LSI determines the relevant incentive parameters $a_{1}$ and $b_{1}$, and then, the LSP selects their combined efforts level $\left(L_{11}, L_{21}\right)$, obtaining the benefits $\pi_{1}$ of phase I. At the end of phase I, according to the actual output $y_{1}$, the LSI judges the operation capability of the LSP in phase I and thus determines the incentive parameters $a_{2}$ and $b_{2}$ of phase II. Then, the LSP selects their combined effort levels $\left(L_{12}, L_{22}\right)$ to form the system output $y_{2}$ and obtain benefit $\pi_{2}$ in phase II.

A two-phase model of LSSC cooperation is constructed, in which the LSI in phase II makes decisions based on the output $y_{1}$ of phase I.

2.2.1. Objective Function and Constraint Condition. As the leader the logistics cooperation in LSSC, the LSI mainly makes decisions of $L_{1 t}, L_{2 t}, a_{t}, b_{t}(t=1,2)$ to maximize their expected benefits, that is, to maximize their deterministic equivalent utility.

The objective function with altruistic properties is as follows: formula (11). At the same time, to ensure that LSP participate in the cooperation, two participation constraints can be expressed as formulas (12) and (13) (Table 3):

$$
\begin{aligned}
\max _{L 1 t, L 2 t, a t, b t} I E_{A}= & E\left[y_{1}-\pi_{1}\left(y_{1}\right)\right]+i E\left[\left(y_{2}-\pi_{2}\left(y_{2}\right)\right)\right] \\
& +\lambda_{A} *\left(E\left[\pi_{1}\left(y_{1}\right)-I\left(L_{11}, L_{21}\right)\right]-\frac{1}{2} \rho \operatorname{var}\left(\pi_{1}+\delta \pi_{2}\right)+i E\left[\pi_{2}\left(y_{2}\right)-I\left(L_{12}, L_{22}\right)\right]\right),
\end{aligned}
$$

s.t. PC1: $I E_{C} \geq u_{1}+u_{2}$,

PC2: $I E_{C 2}=\delta+d\left(I E_{C}+I E_{A 2}\right)$.

2.2.2. Model Equation in Phase II. In phase II, $I E_{C 2}$ and $I E_{A 2}$

in (13) can be further expressed as

$$
\begin{aligned}
I E_{A 2}= & E\left[\left(y_{2}-\pi_{2}\right) \mid y_{1}\right]+\lambda_{A} *\left(E\left[\left(\pi_{2} \mid y_{1}\right)-I\left(L_{12}, L_{22}\right)\right]-\frac{1}{2} \rho \operatorname{var}\left(\pi_{2} \mid y_{1}\right)\right)=-a_{2}+\left(1-b_{2}\right) E\left(y_{2} \mid y_{1}\right) \\
& +\lambda_{A} *\left[a_{2}+b_{2} E\left(y_{2} \mid y_{1}\right)-I\left(L_{12}, L_{22}\right)-\frac{1}{2} \rho \operatorname{var}\left(\pi_{2} \mid y_{1}\right)\right], \\
I E_{C 2}= & E\left[\left(\pi_{2} \mid y_{1}\right)-I\left(L_{12}, L_{22}\right)\right]-\frac{1}{2} \rho \operatorname{var}\left(\pi_{2} \mid y_{1}\right)+\lambda_{c} * E\left[\left(y_{2}-\pi_{2}\right) \mid y_{1}\right]=a_{2}+b_{2} E\left(y_{2} \mid y_{1}\right)-I\left(L_{12}, L_{22}\right) \\
& -\frac{1}{2} \rho \operatorname{var}\left(\pi_{2} \mid y_{1}\right)+\lambda_{c} *\left[-a_{2}+\left(1-b_{2}\right) E\left(y_{2} \mid y_{1}\right)\right] .
\end{aligned}
$$

Under the optimal decision, the LSP maximizes its own benefits by choosing the effort level, that is, to satisfy the twophase incentive compatibility constraint:

$$
\text { ICC - } 1:\left(L_{11}, L_{21}\right) \in \arg \max I E_{C},
$$

$$
\text { ICC - } 2:\left(L_{12}, L_{22}\right) \in \arg \max I E_{C 2} .
$$

LSI with altruism makes decisions based on the output of phase I, while also satisfying the participation constraints of LSP, namely,

$$
\begin{aligned}
& \max _{a_{2}, b_{2}} E\left(y_{2} \mid y_{1}\right)-E\left(\pi_{2} \mid y_{1}\right)+\lambda_{A} *\left(E\left[\left(\pi_{2} \mid y_{1}\right)-I\left(L_{12}, L_{22}\right)\right]-\frac{1}{2} \rho \operatorname{var}\left(\pi_{2} \mid y_{1}\right)\right), \\
& \text { s.t. } I E_{C 2}=E\left[\left(\pi_{2} \mid y_{1}\right)-I\left(L_{12}, L_{22}\right)\right]-\frac{1}{2} \rho \operatorname{var}\left(\pi_{2} \mid y_{1}\right)+\lambda_{c} * E\left[\left(y_{2}-\pi_{2}\right) \mid y_{1}\right] \geq u_{2} .
\end{aligned}
$$


TABLe 3: Model index setting-2.

\begin{tabular}{ccc}
\hline & Index & Index description \\
\hline $\mathrm{PC}_{1}$ & $\begin{array}{c}\text { Deterministic equivalent } \\
\text { benefit }\end{array}$ & $\begin{array}{c}\text { Deterministic equivalent benefit obtained from the entire system by the LSP from participating in the } \\
\text { cooperation is not less than the retained benefit of not participating in the cooperation }\end{array}$ \\
$\mathrm{PC}_{2}$ & $\begin{array}{c}\text { Participation constraint of } \\
\text { LSP }\end{array}$ & Participation constraint of LSP in phase II \\
$d$ & Bargaining power & Share of LSP income from LSSC system in phase II \\
$\delta$ & coefficient & Constant
\end{tabular}

Substituting (19) into (18), the time consistency constraint under the dynamic cooperation is

$$
\begin{aligned}
\mathrm{TL} & : \max _{a_{2}, b_{2}}\left(1+\lambda_{c}\right) E\left(y_{2} \mid y_{1}\right)-\left(1+\lambda_{A}\right) \\
& \cdot\left[I\left(L_{12}, L_{22}\right)-\frac{1}{2} \rho \operatorname{var}\left(\pi_{2} \mid y_{1}\right)\right] .
\end{aligned}
$$

In summary, formulas (11)-(13), (16), (17), and (20) constitute the cross-phase cooperation dynamic incentive model, and from formulas (4)-(6),

$$
\begin{aligned}
\operatorname{var}\left(\pi_{2} \mid y_{1}\right) & =b_{2}^{2}\left(1-\varphi^{2}\right) \sigma^{2}, \\
\operatorname{var}\left(\pi_{1}+\delta \pi_{2}\right) & =\operatorname{var}\left(\pi_{1}\right)+2 \operatorname{cov}\left(\pi_{1}+i \pi_{2}\right)+\operatorname{var}\left(i \pi_{2} \mid x_{1}\right) \\
& =\left[\left(b_{1}+i \varphi b_{2}\right)^{2}+\left(1-2 \varphi^{2}\right) i^{2} b_{2}^{2}\right] \sigma^{2} .
\end{aligned}
$$

\section{Model Deriving and Solving}

According to the hypothesis, the cooperation lasts for two phases; therefore, the output level of the LSP will not affect the subsequent income distribution. So, for a given incentive coefficient, the LSP chooses the level of effort $\left(L_{12}, L_{22}\right)$ with the goal of maximizing the self-return in phase II and passes the first-order condition according to the formula. Using backward induction, the above dynamic model is solved to subgame perfect Nash equilibrium:

$$
\left(L_{12}, L_{22}\right)=\left(\frac{b_{2}\left(\beta_{2}-\varnothing r \sqrt{\beta_{1} \beta_{2}}\right)}{\left(1-r^{2}\right) \beta_{1} \beta_{2}}, \frac{b_{2}\left(\varnothing \beta_{1}-r \sqrt{\beta_{1} \beta_{2}}\right)}{\left(1-r^{2}\right) \beta_{1} \beta_{2}}\right) .
$$

Substituting the formulas (16) and (17) into the formula (15) and substituting the formulas (23) and (25) into the formula (22), the second-order refined Nash equilibrium can be derived from the first-order condition:

$$
\begin{aligned}
& a_{2}=\delta+\left(d-b_{2}\right) E\left(y_{2} \mid y_{1}\right)+(1-d)\left(\frac{1}{2} \beta_{1} \widehat{L}_{12}^{2}+\frac{1}{2} \beta_{2} \widehat{L}_{22}^{2}+r \sqrt{\beta_{1} \beta_{2}} \widehat{L}_{12} \widehat{L}_{22}\right)+\frac{1}{2} \rho(1-d)\left(1-\varphi^{2}\right) b_{2}^{2} \sigma^{2}, \\
& b_{2}=\frac{\rho \beta_{1} \beta_{2} \sigma^{2}\left(1-r^{2}\right)\left(\beta_{1} \varnothing^{2}-2 \varnothing r \sqrt{\beta_{1} \beta_{2}}+\beta_{2}\right)}{\rho \beta_{1} \beta_{2} \sigma^{2}\left(1-\varphi^{2}\right)\left(1-r^{2}\right)+\beta_{1} \varnothing^{2}-2 \varnothing r \sqrt{\beta_{1} \beta_{2}}+\beta_{2}} .
\end{aligned}
$$

Let

$$
\begin{aligned}
D= & \delta+\left(d-b_{2}\right)\left(\widehat{L}_{12}+\varnothing \widehat{L}_{12}\right) \\
& +(1-d)\left(\frac{1}{2} \beta_{1} \widehat{L}_{12}^{2}+\frac{1}{2} \beta_{2} \widehat{L}_{22}^{2}+r \sqrt{\beta_{1} \beta_{2}} \widehat{L}_{12} \widehat{L}_{22}\right) \\
& +\frac{1}{2} \rho(1-d)\left(1-\varphi^{2}\right) b_{2}^{2} \sigma^{2} .
\end{aligned}
$$

Formula (25) turns to

$$
\pi_{2}=D+\left(d-b_{2}\right) E\left(\eta \mid y_{1}\right)+b_{2} y_{2}
$$

Formula (27) shows that, in the dynamic incentive problem of the LSSC, in phase I, the LSP affects the system output through the choice of the effort level of the two types of logistics cooperation tasks, which in turn affects the expectations $E\left(\eta \mid y_{1}\right)$ of the LSI on the logistics operation capabilities of the LSP. Formula (27) also shows that the implicit reputation effect, expressed by $\left(d-b_{2}\right) E\left(\eta \mid y_{1}\right)$, can affect the total income of the phase by adjusting the fixed payment in phase II. Besides the influence of explicit incentives (contract incentives), the income of LSP in phase II is also affected by the expectations of the LSI about the operation ability of LSP. In order to ensure that $d>b_{2}$ is one of the necessary conditions to ensure the effective exertion of implicit reputation effect in dynamic incentive. 
According to the time sequence of the dynamic game, further solve the subgame perfect Nash equilibrium of LSP in phase I. According to the incentive compatibility constraint, substituting $\pi_{2}$ into formula ICC -1 , the firstorder condition for $\left(L_{11}, L_{21}\right)$ is

$$
\left(L_{11}, L_{21}\right)=\left(\frac{b_{1}\left(\beta_{2}-\varnothing r \sqrt{\beta_{1} \beta_{2}}\right)\left[1+i \varphi\left(d-b_{2}\right)\right.}{\beta_{1} \beta_{2}\left(1-r^{2}\right)}, \frac{b_{1}\left(\varnothing \beta_{1}-r \sqrt{\beta_{1} \beta_{2}}\right)\left[1+i \varphi\left(d-b_{2}\right)\right]}{\beta_{1} \beta_{2}\left(1-r^{2}\right)}\right) .
$$

Finally, solve LSI optimal incentive coefficient of phase I. The optimization model is

$$
\max _{a_{t}, \beta_{t}, L_{1 t}, L_{2 t}} E\left(y_{1}\right)-E\left(\pi_{1}\left(y_{1}\right)\right)+i\left[E\left(y_{2}\right)-E\left(\pi_{2}\left(y_{2}\right)\right)\right]
$$

s.t. $I E_{C} \geq u_{1}+u_{2}$.

Substituting formulas (10), (22), (25), and (28) into the above optimization model, the solution is

$$
b_{1}=\frac{\left(\beta_{1} \varnothing^{2}-\varnothing r \sqrt{\beta_{1} \beta_{2}}\right)\left(1-r^{2}\right) \rho \beta_{1} \beta_{2} \varphi \sigma^{2}+1-\operatorname{di\varphi }}{\beta_{1} \varnothing^{2}-2 \varnothing r \sqrt{\beta_{1} \beta_{2}}+\beta_{2}+\rho \beta_{1} \beta_{2} \sigma^{2}\left(1-r^{2}\right)\left(1-\varphi^{2}\right)} .
$$

Considering the different stages of cooperation and the relevance between input costs of the two types of logistics tasks during the operation process, the observability of the output performance of the basic logistics task and the logistics quality assurance task undertaken by the logistics capacity provider is different. These characteristics will affect the incentive efficiency directly in the LSSC cooperation mechanism. Thus, the further derivation is given as follows.
According to formula (28), transform the expressions and derive each other:

$$
\begin{aligned}
& \frac{\partial L_{12}}{\partial L_{22}}=-\frac{r \sqrt{\beta_{1} \beta_{2}}}{\beta_{1}}, \\
& \frac{\partial L_{22}}{\partial L_{12}}=-\frac{r \sqrt{\beta_{1} \beta_{2}}}{\beta_{2}} .
\end{aligned}
$$

According to formula (28) the first order conditions are obtained, respectively:

$$
\begin{aligned}
& \frac{\partial L_{22}}{\partial b_{2}}=\frac{\varnothing \beta_{1}-r \sqrt{\beta_{1} \beta_{2}}}{\left(1-r^{2}\right) \beta_{1} \beta_{2}}, \\
& \frac{\partial L_{12}}{\partial b_{2}}=\frac{\beta_{2}-\varnothing r \sqrt{\beta_{1} \beta_{2}}}{\left(1-r^{2}\right) \beta_{1} \beta_{2}} .
\end{aligned}
$$

According to formulas (25) and (30), the incentive coefficients of the two stages are $b_{1}$ and $b_{2}$ derived from the task correlation coefficient $r$, respectively:

$$
\begin{aligned}
& \frac{\partial b_{1}}{\partial r}=\frac{\left(\beta_{1} \varnothing^{2}+\beta_{2}-2 \varnothing r \sqrt{\beta_{1} \beta_{2}}+1-i \varphi d\right)\left(1-r^{2}\right)(1+i \varphi)\left(\beta_{1} \varnothing-r \sqrt{\beta_{1} \beta_{2}}\right) \sigma^{2} \beta_{1}^{2} \beta_{2}^{2} r}{\left[\beta_{1} \varnothing^{2}+\beta_{2}-2 \varnothing r \sqrt{\beta_{1} \beta_{2}}+\rho \beta_{1} \beta_{2} \sigma^{2}\left(1-r^{2}\right)\left(1-\varphi^{2}\right)\right]^{2}}, \\
& \frac{\partial b_{2}}{\partial r}=\frac{\sigma^{2} \beta_{1}^{2} \beta_{2}^{2} r\left(1-r^{2}\right)\left(\beta_{1} \varnothing-r \sqrt{\beta_{1} \beta_{2}}\right)}{\left[\beta_{1} \varnothing^{2}+\beta_{2}-2 \varnothing r \sqrt{\beta_{1} \beta_{2}}+\rho \beta_{1} \beta_{2} \sigma^{2}\left(1-r^{2}\right)\left(1-\varphi^{2}\right)\right]^{2}} .
\end{aligned}
$$

According to formulas (25) and (30), two stage incentive coefficients are $b_{1}$ and $b_{2}$ derived from $\rho$, respectively:

$$
\frac{\partial b_{1}}{\partial \rho}=-\frac{\delta \varphi \sigma^{2} \beta_{1}^{2} \beta_{2}^{2}(1-d)\left(1-r^{2}\right)\left(\beta_{1} \varnothing^{2}+\beta_{2}-2 \varnothing r \sqrt{\beta_{1} \beta_{2}}\right)}{\left[\beta_{1} \varnothing^{2}+\beta_{2}-2 \varnothing r \sqrt{\beta_{1} \beta_{2}}+\rho \beta_{1} \beta_{2} \sigma^{2}\left(1-r^{2}\right)\left(1-\varphi^{2}\right)\right]^{2}},
$$




$$
\frac{\partial b_{2}}{\partial \rho}=-\frac{\sigma^{2} \beta_{1}^{2} \beta_{2}^{2}\left(1-r^{2}\right)\left(\beta_{1} \varnothing^{2}+\beta_{2}-2 \varnothing r \sqrt{\beta_{1} \beta_{2}}\right)^{2}}{\left[\beta_{1} \varnothing^{2}+\beta_{2}-2 \varnothing r \sqrt{\beta_{1} \beta_{2}}+\rho \beta_{1} \beta_{2} \sigma^{2}\left(1-r^{2}\right)\left(1-\varphi^{2}\right)\right]^{2}}
$$

\section{The Equilibrium Analyzing}

4.1. Comparison of the Two Types of Effort $\left(\left(\mathbf{L}_{11}, \mathbf{L}_{21}\right)\right.$ and $\left.\left(\mathbf{L}_{12}, \mathbf{L}_{22}\right)\right)$ at Different Phases of Cooperation. By comparing the effort $\left(L_{11}, L_{21}\right)$ of LSP in phase I with the effort $\left(L_{12}, L_{22}\right)$ in phase II, we can find that the LSP effort of the two types of cooperation in phase $\mathrm{I}$ is still affected by $1-i \varphi\left(d-b_{2}\right)$. Assume that the incentive coefficients $b_{t}$ of each phase are equal; if $d>b_{2}$, then $L_{11}>L_{12}$ and $L_{21}>L_{22}$; else if $d<b_{2}$, then $L_{11}<L_{12}$ and $L_{21}<L_{22}$, which shows that, under the condition that the reputation effect played a role, that is, $d>b_{2}$, the LSP's effort level in phase I is significantly improved. $i \varphi d$ can be regarded as the reputation effect, and $i \varphi b_{2}$ is the ratcheting effect. When $i \varphi d>i \varphi b_{2}$, the effort of the LSP will be significantly improved.

Reputation incentives, as a supplement of explicit incentives, can help to form an effective incentive mechanism which integrated short-term incentives with long-term incentives.

In the long run, with increasing cooperation complexity, this incentive model can play an important role to reduce the short-term behavior of LSP, maintain stability of cooperation, and get more profit.

4.2. Comparison of Two Types of Cooperation Effort $\mathbf{L}_{12}$ and $\mathrm{L}_{22}$ under Task Correlation. According to the multitask relevance, we can find the following:

(1) If $r \in(0,1]$, the two types of cooperation tasks have an alternative relationship. (i) According to formulas (31) and (32), $\beta_{1}$ unit change in investment in valueadded cooperation by LSP will lead to a change in $r \sqrt{\beta_{1} \beta_{2}}$ unit in regular logistics tasks. (ii) According to the output function $y_{t}=\theta+L_{1 t}+\varnothing L_{2 t}+\varepsilon_{t}$, when the decrease in system output $r \sqrt{\beta_{1} \beta_{2}} / \beta_{1}$ caused by the decreasing input of regular logistics task is less than the increase in system output $\varnothing$ caused by the increase in value-added logistics tasks, that is, $\left(r \sqrt{\beta_{1} \beta_{2}} / \beta_{1}\right)<\varnothing$, the output of the system increases. At this time, LSP will choose to increase input in value-added logistics task to increase system output. If $\left(r \sqrt{\beta_{1} \beta_{2}} / \beta_{1}\right)>\varnothing$, LSP will invest more on regular logistics service cooperation tasks.

(2) If $r \in[-1,0)$, one unit increase of investment in value-added cooperation by LSP will lead to an increase in $r \sqrt{\beta_{1} \beta_{2}} / \beta_{1}$ unit in regular logistics tasks. In this situation, the LSP will make the decision to increase the investment in the both the types of cooperation tasks.

In the same way, similar conclusions can be drawn for phase I.
4.3. The Effect of the Uncertainty of Output $\left(\sigma_{1}^{2}, \sigma_{2}^{2}\right)$ on the Dynamic Incentive Intensity $\left(\mathbf{b}_{1}, \mathbf{b}_{2}\right)$. The increase in the variance of the observable variables of the input results means that the input of the LSP in the regular cooperation task is less relevant to the final output of the task. Regular logistics cooperation tasks are relatively easy to observe and control, so $\sigma_{1}^{2}$ is limited. For the second type of value-added task, the outcome of the input is relatively difficult to observe. When $\sigma_{2}^{2} \longrightarrow+\infty$, the value-added task by LSP can hardly produce direct benefits in a short period of time. From formulas (30) and (25),

$$
\begin{aligned}
\lim _{\sigma_{1}^{2} \longrightarrow \infty} b_{1} & =\frac{\left(\beta_{1} \varnothing^{2}-\varnothing r \sqrt{\beta_{1} \beta_{2}}\right)\left(1-r^{2}\right) \rho \beta_{1} \beta_{2} \varphi \sigma^{2}+1-d i \varphi}{\beta_{1} \varnothing^{2}-2 \varnothing r \sqrt{\beta_{1} \beta_{2}}+\beta_{2}+\rho \beta_{1} \beta_{2} \sigma^{2}\left(1-r^{2}\right)\left(1-\varphi^{2}\right)}, \\
\lim _{\sigma_{2}^{2} \longrightarrow \infty} b_{2} & =0 .
\end{aligned}
$$

As for the first type of regular cooperation task where the input result is easy to observe, the incentive intensity of the LSI decreases with the increase in the uncertainty $\sigma_{1}^{2}$. As for the second type of cooperative tasks whose results are not easy to observe, the incentive coefficient $b_{2}$ approaches 0 , indicating that it is difficult to encourage the LSP investment effectively.

4.4. The Impact of Task Relevance $\mathbf{r}$ on Dynamic Incentive Intensity $\left(\mathbf{b}_{1}, \mathbf{b}_{2}\right)$. According to formulas (34) and (35), we can find that because $r \in[-1,1]$, it is easy to prove that $\beta_{1} \varnothing^{2}-2 \varnothing r \sqrt{\beta_{1} \beta_{2}}+\beta_{2} \geq 0$, so whether the above two formulas are positive or negative is determined by $\left(\beta_{1} \varnothing-\varnothing r \sqrt{\beta_{1} \beta_{2}}\right)$.

(1) When the two types of logistics cooperation tasks have an alternative relationship $(0<r<1)$, the above two formulas are both positive. According to the comparison of the input of the two types of cooperation under the task correlation, when $\left(r \sqrt{\beta_{1} \beta_{2}} / \beta_{1}\right)<\varnothing$, the input of regular logistics service cooperation task is reduced, the input of valueadded logistics service cooperation task will increase, and the output of the entire logistic service supply chain system will increase. That is to say, when the logistics tasks have a substitution relationship, when the value-added logistics service cooperation task contributes more significantly to the overall performance, as the task substitution relationship changes the strength ( $\rho$ increases), the two-phase incentive coefficients $b_{1}$ and $b_{2}$ will increase. At the same time, the LSI is more inclined to motivate LSP to increase investment in value-added logistics 
service cooperation task to obtain higher system benefits.

When $\left(r \sqrt{\beta_{1} \beta_{2}} / \beta_{1}\right)>\varnothing$, the regular logistics service cooperation task contributes more significantly to overall performance, the LSI will tend to motivate LSP to increase investment in regular logistics service cooperation task to achieve higher system benefits.

(2) When the two types of logistics tasks have a complementary relationship, because $-1<r<0$, then $\left(r \sqrt{\beta_{1} \beta_{2}} / \beta_{1}\right)<\varnothing$, so both $\partial b_{1} / \partial r$ and $\partial b_{2} / \partial r$ are less than zero. Due to the complementary relationship between the two types of logistics tasks, the LSI will encourage LSP to increase the level of effort for the two types of logistics tasks at the same time. As the complementary relationship strengthens ( $\rho$ decreases), $b_{1}$ and $b_{2}$ will increase.

(3) Further comparing formula (34) with (35), when $\varnothing$ is large enough, $\left(\partial b_{1} / \partial r\right)>\left(\partial b_{2} / \partial r\right)$, it means that when the value-added logistics service cooperation task has a large enough impact on the performance of the logistic service supply chain system, the incentive coefficients of the various cooperation phases are more sensitive to the changes in the task correlation coefficient, indicating that the cooperation phases are interrelated, and value-added logistics service cooperation task not only has a significant impact on the system performance at this phase but also has an impact on the system performance in the subsequent cooperation phase, thereby amplifying the response of the incentive coefficient to the task correlation coefficient. In practice, the construction of valueadded infrastructure that plays an important role in the overall operation has a significant role in improving the performance of each phase of operation. The LSI should pay full attention to the investment in this type of logistics service cooperation.

\subsection{The Effect of Incentive Coefficient $\mathbf{b}_{2}$ on Effort Level $\left(\mathbf{L}_{12}, \mathbf{L}_{22}\right)$}

(1) When $r \in(0,1]$ which means the two types of logistics tasks have a complementary relationship, (1) if $\left(r \sqrt{\beta_{1} \beta_{2}} / \beta_{1}\right)<\varnothing$, then the value-added logistics service task input level $e_{22}$ increases with the increase in the incentive coefficient $b_{2} ;(2)$ if $\left(r \sqrt{\beta_{1} \beta_{2}} /\right.$ $\left.\beta_{1}\right)>\varnothing$, the value-added logistics service task input level decreases with the increase in the incentive coefficient; (3) if $\left(\beta_{2} / r \sqrt{\beta_{1} \beta_{2}}\right)>\varnothing$, then the level of routine logistics service task investment increases with the increase in the incentive coefficient; and (4) if $\left(\beta_{2} / r \sqrt{\beta_{1} \beta_{2}}\right)<\varnothing$, the level of routine logistics service task investment decreases with the increase in the incentive coefficient. In all, the above analysis shows that, due to the substitution relationship between logistics service tasks, the impact of incentive coefficients on different logistics tasks will be different due to the difference in the impact of the two substitutions transformation on the final output.
(2) When the two types of tasks have a complementary relationship, for $r \in[-1,0)$, both $\partial L_{22} / \partial b_{2}$ and $\partial L_{12} / \partial b_{2}$ are greater than zero. As LSI increases the incentive coefficient, LSP will increase the level of investment in the two logistics cooperation tasks at the same time.

According to the above analysis, under the combined effect of the characteristics of logistics service cooperation and the difference in the impact of different services on overall performance, the difference in the incentive effect of the LSI is more significant: when $\left(r \sqrt{\beta_{1} \beta_{2}} / \beta_{1}\right)>\varnothing$, the incentives of the LSI are more effective in regular logistics task; when $\left(\beta_{2} / r \sqrt{\beta_{1} \beta_{2}}\right)<\varnothing$, the incentives of the LSI are more on the input of value-added logistics tasks; and when $\left(r \sqrt{\beta_{1} \beta_{2}} / \beta_{1}\right)<\varnothing<\left(\beta_{2} / r \sqrt{\beta_{1} \beta_{2}}\right)$, the incentives of the LSI will act on two types of logistics cooperation at the same time. In the practice of logistic service supply chain, the LSI should pay attention to the effect of different motivation factors on motivation efficiency, effectively identify the specific content and characteristics of patient needs, and design incentive strategies that meet the incentive compatibility to effectively play the role of motivation.

4.6. The Relationship between Risk Aversion Degree $\rho$, Operational Capability Expectation $\varphi$, and Incentive Coefficients $\mathbf{b}_{1}, \mathbf{b}_{2}$. According to formulas (36) and (37), we can find the following.

As $\rho \in[-1,1],\left(\partial b_{1} / \partial \rho\right) \leq 0,\left(\partial b_{2} / \partial \rho\right) \leq 0$, so in the logistic service supply chain cooperation, as the degree of risk aversion in LSI increases, the LSI will reduce the incentive intensity at each phase. In practice, LSI should choose LSI with different risks according to the extend of different tasks affected by external factors. The same can be proved: $\left(\partial b_{1} / \partial \rho\right) \geq 0$ and $\left(\partial b_{1} / \partial \rho\right) \geq 0$, so as the uncertainty of community operation ability increases, the LSI will increase the incentive intensity at each phase. According to the hypothesis of the paper, the expected coefficient of logistics service operation ability reflects the inclusion of relevant LSI in the final system output information on operational capabilities. For integrated logistics services that require high operational capabilities, LSI faces increased risks when external uncertainties increase. In order to increase the enthusiasm of LSI, the LSI will significantly increase the incentive intensity.

\section{Conclusion}

In the principal-agent relationship between SSI and LSP in LSSC, a great number of researches focus on the mechanism of regular cooperation tasks of logistics activities. However, with the deepening of cooperation, LSP is usually required to do more tasks to achieve value-added goals. Due to the significant differences in performance in different tasks when LSP makes the same effort and some kinds of correlation between LSP's input cost in different tasks, the incentive efficiency of the entire LSSC is weakened and optimal system performance cannot be obtained. So, based on the principal-agent model, this paper considered the 
dynamic incentive problem of multitask cooperation in LSSC, and the conclusion is as follows:

(1) The implicit reputation effect in dynamic cooperation can effectively improve system incentive efficiency [20] and realize an effective incentive model that combines long-term incentives and short-term incentives.

(2) Factors, such as the relevance of the cooperation phase in the dynamic cooperation, the relevance of multiple logistics tasks, and the difference in the contribution of different logistics cooperation to the performance of the LSSC, have a significant impact on the incentive effect.

(3) When the two kinds of cooperation tasks have complementary relationships, both the LSP's choice of logistics tasks and the incentives of the LSI will simultaneously act on the two types of cooperation tasks. When the tasks have an alternative relationship, the LSI will choose the cooperation task that is more conducive to improving system performance, and the LSI's incentive policy will also vary due to the differences in the impact of different cooperation on system performance.

(4) The antirisk ability of LSI and the uncertainty of the operating environment will also have a certain impact on the incentive effect of the LSSC. Therefore, the LSI should comprehensively consider the integration requirements of logistics cooperation and the risk tolerance of LSI when choosing a cooperative enterprise.

Currently, the vertical cooperation of LSSC has become an important research field of the logistics service supply chain, and the problems will be increasingly complicated in practice. The paper mainly discusses the dynamic incentive mechanism of the vertical cooperation of LSSCs from the management model. The research lacks further empirical tests based on related research. The next step is to conduct a more in-depth study on the implementation and performance of specific mechanisms from an empirical perspective.

With global supply chain reconstruction, the vertical cooperation of LSSC has become a new normalization phenomenon. The paper studies the incentive mechanism of multitask cooperation in logistics service supply chain (LSSC) by building a dynamic incentive model. This paper is limited to static cooperation, and the next step is to consider the temporal and spatial complexity of LSSC and the dynamic characteristics of upstream and downstream cooperation. In the multistage dynamic cooperation case, specific cooperation mechanisms and behavioral strategies should be studied.

\section{Data Availability}

The data in this paper are obtained by simulation.

\section{Conflicts of Interest}

The authors declare that they have no conflicts of interest.

\section{Acknowledgments}

This work was supported by the National Natural Science Foundation of China (Research on a Digital InnovationDriven Entrepreneurship Model of New Ventures, Grant no. 72091313) and Base of International Science and Technology Innovation and Cooperation on Big Data Technology and Management (Grant no. 2018WK4030).

\section{References}

[1] P. J. Agrell, R. Lindroth, and A. Norrman, "Risk, information and incentives in telecom supply chains," International Journal of Production Economics, vol. 90, no. 1, pp. 1-16, 2004.

[2] T. Guofeng, D. Bin, and S. Han, "Research on the incentive mechanism for multi-task moral hazard in application service outsourcing," Systems Engeering: Theory \& Practive, vol, vol. 33, no. 5, pp. 1175-1184, 2013.

[3] Ge. Caihong and Ji. Bifa, "Research on multi-task cooperation mechanism of logistics service supply chain," ournal of Zhejiang Shuren University, vol. 18, no. 1, pp. 53-59, 2018.

[4] A. H. L. Lau, H.-S. Lau, and Y.-W. Zhou, "Considering asymmetrical manufacturing cost information in a twoechelon system that uses price-only contracts," IIE Transactions, vol. 38, no. 3, pp. 253-271, 2006.

[5] Y. H. Albert and S. L. Tong, "Contracting and information sharing under supply chain competition," Informs, vol. 4, pp. 701-715, 2008.

[6] M. Ngyuyen and J. C. Poundon, "Optimal resource extraction contract with adverse selection," Resources Policy, vol. 3l, no. 2, pp. 78-85, 2006.

[7] H. Krishnan and R. A. Winter, "Inventory dynamics and supply chain coordination," Management Science, vol. 56, no. 1, pp. 141-147, 2010.

[8] L. Liming and S. Weixin, "Dynamic competitive newsvendors with service-sensitive demands," Manufacturing and Service Operation Management, vol. 9, no. 1, pp. 84-93, 2007.

[9] F. Bernstein and A. Dedergruen, "Coordination mechanisms for supply chains under price and service competition," Manufacturing \& Service Operations Management, vol. 9, no. 3, pp. 243-262, 2007.

[10] M. R. Frascatore, F. Mahmoodi, and F. Mahmoodi, "Longterm and penalty contracts in a two-stage supply chain with stochastic demand," European Journal of Operational Research, vol. 184, no. 1, pp. 147-156, 2008.

[11] W. Qing, D. Bin, Q. Yu et al., "Third party logistics coordinating contracts for low value perishable products with loss dependent on logistics effort levels," Journal of Management Sciences in China, vol. 17, no. 12, pp. 15-26, 2014.

[12] F. Yingying, L. Jiejiao, and C. Ruonan, "Evolution mechanism of enterprise financing innovation in open collaborative innovation system analysis of multiple evolutionary game models based on knowledge sharing," Inquiry Into Economic Issues, vol. 9, no. 458, pp. 175-184, 2020.

[13] B. S. Desgagne, "How to restore higher-powered incentives in multitask agencies," The Journal of Low, Economy and Organization, vol. 5, pp. 121-129, 1999.

[14] Y. Yiding and H. Daqing, "Research on incentive and supervision mechanism of open innovation based on evolutionary game," Yunnan Social Sciences, vol. 2, pp. 75-79, 2016.

[15] Y. Wang, W. P. Tay, and W. Hu, "Multitask Diffusion LMS with Optimized Inter-cluster Cooperation," in Proceedings of the 2016 IEEE Statistical Signal Processing Workshop (SSP), IEEE, Palma de Mallorca, Spain, 2016. 
[16] Y. Wang, W. P. Tay, and W. Hu, "A multitask diffusion strategy with optimized inter-cluster cooperation," IEEE Journal of Selected Topics in Signal Processing, vol. 11, no. 3, pp. 504-517, 2017.

[17] M. . c. Weitman, "Efficicent incentive contracts," The Quarterly Journal of Economics, vol. 17, no. 6, pp. 11-23, 1980.

[18] B. Holmstrom and P. Milgrom, "Aggregation and linearity in the provision of inter-temporal incentives," Econometrical, vol. 17, no. 2, pp. 15-26, 1987.

[19] C. Yanfeng and H. Pengfei, "Research on incentive of knowledge transfer in cooperative R \& D outsourcing under open innovation--based on evolutionary game model," Research on Science and Technology Management, vol. 39, no. 423, pp. 143-151, 2019.

[20] V. C. Gogineni and M. Chakraborty, "“Improving the performance of multitask diffusion APA via controlled intercluster cooperation", circuits and systems I: regular papers," IEEE Transactions on, vol. 99, pp. 1-10, 2019. 